\title{
Serum Levels of ITGBLI as an Early Diagnostic Biomarker for Hepatocellular Carcinoma with Hepatitis B Virus Infection
}

Fei Ye, (D) ${ }^{1, *}$ Wei Huang, ${ }^{2, *}$ Yuan Xue, ${ }^{3, *}$ Erjiang Tang, ${ }^{4,5}$ Mingjie Wang, ${ }^{6}$ Fengchun Shi, (D)' Dong Wei, ' Yue Han, ' Peizhan Chen, ${ }^{7}$ Xinxin Zhang,' Demin Yu'

'Department of Infectious Diseases, Research Laboratory of Clinical Virology, Ruijin Hospital, Shanghai Jiaotong University, School of Medicine, Shanghai, 200025, People's Republic of China; ${ }^{2}$ Department of Gastroenterology, Affiliated Hospital of Nantong University, Nantong, 226000, People's Republic of China; ${ }^{3}$ Institute of Hepatology, The Third People's Hospital of Changzhou, Changzhou, 213000 , People's Republic of China; ${ }^{4}$ Center for Clinical Research and Translational Medicine, Yangpu Hospital, Tongji University School of Medicine, Shanghai, 200090, People's Republic of China; ${ }^{5}$ Institute of Gastrointestinal Surgery and Translational Medicine, Tongji University School of Medicine, Shanghai, 200090, People's Republic of China; ${ }^{6}$ Department of Gastroenterology \& Hepatology, Ruijin Hospital, Shanghai Jiaotong University, School of Medicine, Shanghai, 20182I, People's Republic of China; ${ }^{7}$ Clinical Research Center, Ruijin Hospital, Shanghai Jiao Tong University School of Medicine, Shanghai, 20182I, People's Republic of China

*These authors contributed equally to this work

Correspondence: Demin Yu Email ydmII367@rjh.com.cn

Xinxin Zhang

Email zhangx@shsmu.edu.cn
Purpose: Early diagnostic biomarkers of hepatocellular carcinoma (HCC) are needed to distinguish hepatitis B virus (HBV) associated HCC (HBV-HCC) patients from at-risk patients. We assessed the diagnostic values of serum Integrin beta-like 1 (ITGBL1) for earlystage HBV-HCC.

Patients and Methods: We recruited 716 participators including 299 in the training and 417 in the validation stage, (HBV-HCC, chronic hepatitis B (CHB), HBV-related liver cirrhosis (HBV-LC), and healthy controls) between 2017 and 2020 from three centers. Serum ITGBL1 was measured by ELISA. Receiver operating characteristic (ROC) was used to calculate diagnostic accuracy.

Results: The serum levels of ITGBL1 in HBV-HCC patients were significantly lower than those in CHB and HBV-LC patients. This result was confirmed in the follow-up patients who progressed from HBV-LC to HCC. The optimum diagnostic cutoff value of serum ITGBL1 was $47.93 \mathrm{ng} / \mathrm{mL}$ for detection of early-stage HBV-HCC. The serum ITGBL1 has higher diagnostic accuracy than AFP20 in differentiating the early-stage HBV-HCC from the at-risk patients (area under curve [AUC] 0.787 vs $0.638, \mathrm{p}<0.05)$. For AFP-negative $(<20 \mathrm{ng} / \mathrm{mL})$ HBV-HCC patients, serum ITGBL1 maintained diagnostic accuracy (training cohort: AUC $0.756,95 \%$ confidence interval [CI] $0.683-0.819$, sensitivity $68.18 \%$, and specificity $68.85 \%$; validation cohort: $0.744,0.686-0.796,81.13 \%$, and 55.88\%). Combination ITGBL1 with AFP20 significantly increased diagnostic accuracy in differentiating the HBVHCC from at-risk patients (AUC 0.840; 0.868) than ITGBL1 (AUC 0.773, p <0.05; 0.732, $\mathrm{p}<0.0001$ ) or AFP20 (AUC 0.705, $<<0.0001 ; 0.773$, $<<0.0001$ ) alone.

Conclusion: The serum level of ITGBL1 improved identification of AFP-negative HBVHCC patients, and increased diagnostic accuracy with AFP20 together in the early detection of HBV-HCC.

Keywords: hepatocellular carcinoma, ITGBL1, biomarker, chronic hepatitis B, HBV-related liver cirrhosis

\section{Introduction}

Globally, hepatocellular carcinoma (HCC) is the fourth principal cause of death from cancer, ${ }^{1,2}$ as the most common subtype of liver cancer, and accounts for approximately $85-90 \%$ of all primary liver malignancies. ${ }^{3}$ Although the pathogenesis of HCC is still not clear, hepatitis B virus (HBV) infection and liver cirrhosis remain the major contributors to HCC comprising three main stages in the progression of chronic liver disease. ${ }^{3,4}$ Detection of early-stage HBV-HCC is vital to reduce disease-related mortality in at-risk populations, because patients would 
have a higher survival rate from early tumour resection, ${ }^{5}$ liver transplantation, ${ }^{6}$ or tumour ablation. ${ }^{7}$

Some surveillance strategies such as ultrasonography (US) and serum $\alpha$-fetoprotein (AFP) tests at 6-month intervals were recommended by guidelines ${ }^{8}$ for at-risk individuals, ie those that are serum hepatitis B virus surface antigen (HBsAg)-positive and/or have cirrhosis. However, these routine HCC screenings often have low sensitivity for the detection of early-stage HCC. The effectiveness of US is limited by tumour size $\mathrm{s}^{9,10}$ or in patients with obesity, and AFP, the most commonly used tumour marker, has a suboptimal performance in certain pathological conditions such as chronic liver diseases, germ cell tumours, and gastric cancer. ${ }^{11,12}$ Therefore, there is an urgent need for new serum biomarkers to improve the effectiveness of the early diagnosis in patients with HBV-HCC.

Integrin beta-like 1 (ITGBL1) is a secretory glycoprotein containing ten epidermal growth factor-like repeat domains, and its structure is similar to that of integrin $\beta .^{13,14}$ In recent years, ITGBL1 has been extensively studied as a novel tumour suppressor gene that contributes to tumorigenesis in numerous cancers, such as ovarian cancer, ${ }^{15}$ breast cancer, ${ }^{16}$ and gastrointestinal cancers including colon ${ }^{17,18}$ and stomach cancer. ${ }^{19,20}$ In previous studies, it was found that ITGBL1 is significantly overexpressed in liver fibrosis or cirrhosis tissues associated with HBV infection. ${ }^{21,22}$ Recently, we also showed that ITGBL1 plays an important role in the progression of liver cancer disease, including promoting cell proliferation, metastasis, and invasion of liver cancer cells. ${ }^{23}$ Thus, we hypothesised that serum ITGBL1 might be a promising novel biomarker for the diagnosis and progression of $\mathrm{HBV}$-associated HCC (HBV-HCC) from at-risk individuals (patients with chronic $\mathrm{HBV}$ infection [CHB] and those with HBV-related cirrhosis [HBV-LC]).

Therefore, we designed this study to identify a new serum protein biomarker in HBV-HCC by enrolling 716 participants from three clinic centres. We evaluated and validated the serum ITGBL1 levels for distinguishing HBV-HCC from at-risk individuals, as well as earlystage HBV-HCC from at-risk subjects, and compared its performance with that of AFP.

\section{Patients and Methods}

\section{Patients and Diagnosis}

To form the training cohort, participants were recruited from Ruijin Hospital (Shanghai, China) between
May 2017 and March 2020 and divided into four groups: healthy controls (HC), patients with $\mathrm{CHB}$, patients with HBV-LC, and patients with HBV-HCC. The validation cohort was recruited between April 2019 and September 2020 from the Third People's Hospital of Changzhou (Changzhou, China) and the Shanghai Yangpu Hospital (Shanghai, China). The HC group comprised healthy volunteers with normal liver biochemistry, no viral hepatitis, and no history of liver or other malignant diseases as confirmed by a physical examination at the physical examination centre of Ruijin Hospital. According to the American Association for the Study of Liver Diseases (AASLD) guidelines, CHB was defined as the persistent presence of $\mathrm{HBsAg}$ for more than 6 months. ${ }^{24}$ Liver cirrhosis was diagnosed based on clinical, biochemical, imaging (splenomegaly, blunt angle, and morphological changes), and/or histological examinations, which was described in detail in the Supplementary data. According to 2018 Practice Guidance by the American Association for the Study of Liver Diseases, a majority of HBV-HCC patients were diagnosed by histopathological examination when biopsy and surgical liver specimens were available. The diagnosis of the remaining HBV-HCC patients was established from imaging and serological examinations including ultrasonography, computed tomography scan, hepatic angiography, and serum AFP levels in patients with $\mathrm{HBV}$ infection, in the absence of any alcoholic liver disease or other virus infection. ${ }^{25}$

The study protocol was approved by the ethics committee of each hospital in accordance with the Declaration of Helsinki. Informed consent was obtained from each patient for the use of their blood tests and clinical records.

\section{Clinical Data Collection}

All research data collected included age, sex, serum albumin level, total bilirubin level, alanine aminotransferase (ALT), aspartate aminotransferase (AST), gammaglutamyl transferase (GGT) prothrombin time, platelet count, white blood cells, HBsAg, HBV envelope antigen (HBeAg), AFP, tumour number, and tumour size. The Child-Pugh score was assessed to categorise CHB, HBVLC, and HBV-HCC patients into Child-Pugh grades A, B, or $\mathrm{C}$. The tumour stage of patients with early-stage HBVrelated $\mathrm{HCC}$ was determined according to the following tumour staging systems: the Milan criteria, ${ }^{26}$ the TNM staging by the American Joint Committee on Cancer, ${ }^{27}$ and the Barcelona Clinic Liver Cancer(BCLC) staging criteria. $^{28,29}$ 


\section{Serum Sample Collection, Storage, and Measurements}

Peripheral blood samples from all patients were collected at the time of diagnosis. Blood samples were separated by centrifugation and frozen at $-80^{\circ} \mathrm{C}$ until tested. Serum concentrations of ITGBL1 were measured using a commercially available sandwich ELISA according to the manufacturer's recommendations (Human ITGBL1 ELISA KIT, Jiangsu Jingmei Biological Technology Co, Ltd.). All measurements were performed independently by the laboratory personnel who were blinded to the diagnosis and other clinical information of the participants, and each sample was assayed in duplicate.

\section{Statistical Methods}

All statistical analyses were performed using SPSS for Windows (version 24) and MedCalc software (version 15.2.2). For clinical characteristics, normally distributed continuous variables were presented as mean \pm standard deviation, as median (interquartile range, Q25-Q75) for non-normally distributed continuous data, or as actual values (percentages) for categorical data. All continuous variables were checked for their distributions using the Kolmogorov-Smirnov normality test. Continuous variables with normal distributions were compared using the unpaired Student's $t$-test and analysis of variance. If the data were not normally distributed, a non-parametric test was used, including the Mann-Whitney $U$-test (two subgroups) or Kruskal-Wallis test (three or more). Binary logistic regression analysis was applied to determine the best equation for probability prediction of HBV-HCC from CHB and HBV-LC. We compared serum ITGBL1 levels between HBV-HCC and HBV-LC stages using the paired $t$-test. The diagnostic values of serum ITGBL1 were assessed by calculating the area under the receiver operating characteristic (ROC) curve (AUC), sensitivity, and specificity. To evaluate the combined diagnostic accuracy of ITGBL1 and AFP, binary logistic regression was used to predict the probability of HCC. P-values were twosided, and values less than 0.05 were considered statistically significant.

\section{Results \\ Clinical Characteristics of the Study Population}

According to the selection criteria, 716 participants were included in this study, 299 in the training stage and 417 in the validation stage (Figure 1). The demographic and pathologic features of these participants in the training and validation cohorts are shown in Table 1. The participants were divided into four groups: healthy control, chronic HBV infection, HBV-related cirrhosis, and HBVrelated HCC. As shown in Table 1, males (84.91\%) were predominant in HBV-related liver cancer in the training cohort. Their mean age was $57 \pm 10$ years, the median serum AFP level was $62.22 \mathrm{ng} / \mathrm{mL}$ (interquartile range, 8.74-619.30 ng/mL), $44(41.51 \%)$ patients were AFPnegative ( $<20 \mathrm{ng} / \mathrm{mL}), 22(20.75 \%)$ patients were HBeAgpositive, and $92(86.79 \%)$ patients had liver cirrhosis. According to the Child-Pugh classification, 68 (64.15\%) patients were classified as class A, $32(30.19 \%)$ as class B, and $6(5.66 \%)$ as class $\mathrm{C}$. The proportions of BCLC stages were as follows: stage A 51 (48.11\%) patients, stage B 21 (19.81\%), stage C-D 33 (31.13\%), and missing 1 (0.94\%). For the validation cohort, males $(82.71 \%)$ were still predominant in HBV-related liver cancer. Their mean age was $59 \pm 9$ years, $53(39.85 \%)$ patients were AFP-negative $(<20$ $\mathrm{ng} / \mathrm{mL}$ ), 35 (26.31\%) patients were HBeAg-positive, and $93(69.92 \%)$ patients had liver cirrhosis. We also observed that $65(48.87 \%)$ patients were classified as stage A, 27 $(20.30 \%)$ as stage B, and $35(26.32 \%)$ as stage C-D by the BCLC staging systems. The clinical characteristics of the participants did not differ significantly between the training and validation cohorts (Table S1).

\section{Serum ITGBLI Levels are Downregulated in HBV-HCC Patients Compared to Those in the At-Risk Group}

We first investigated changes in serum ITGBL1 levels in the progression of liver disease from $\mathrm{HC}, \mathrm{CHB}$, and HBVLC to HBV-HCC. It was unexpected that the serum level of ITGBL1 in the HBV-HCC group (42.89 [22.89-63.09] ng/ $\mathrm{mL}$ in the training cohort; $44.61[25.37-62.70] \mathrm{ng} / \mathrm{mL}$ in the validation cohort) was significantly downregulated compared to those in the at-risk groups (CHB, 78.08 [54.68-106.5] $\mathrm{ng} / \mathrm{mL}$ in the training cohort; 66.40 [48.9392.07] $\mathrm{ng} / \mathrm{mL}$ in the validation cohort) and HBV-LC, 64.51 [48.59-93.51] ng/mL in the training cohort; 69.04 [45.76$87.95] \mathrm{ng} / \mathrm{mL}$ in the validation cohort; $\mathrm{P}<0.0001$ ), whereas no significant difference was observed in comparison to that in $\mathrm{HC}(41.63$ [23.81-58.60] $\mathrm{ng} / \mathrm{mL}$ in the training cohort; 43.08 [22.13-57.11] $\mathrm{ng} / \mathrm{mL}$ in the validation cohort; $\mathrm{P}>0.99$ ). The ITGBL1 serum levels did not differ significantly between CHB and HBV-LC groups but were both 


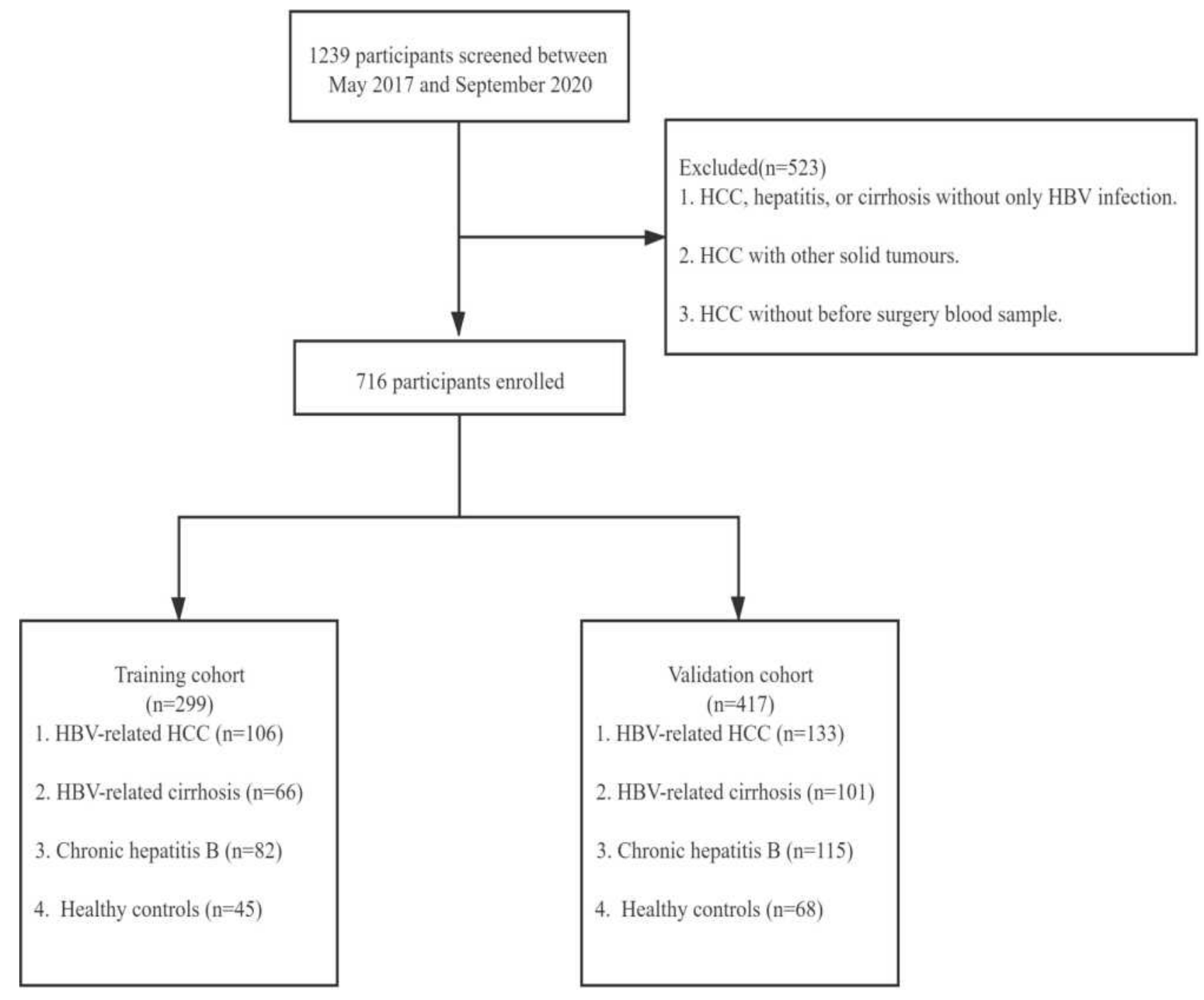

Figure I Study design. Participants of the training stage were collected from Ruijin Hospital. Participants of the validation stage were collected from Yangpu Hospital and the Third People's Hospital of Changzhou.

significantly higher than that in $\mathrm{HC}$ as expected $(\mathrm{P}<0.0001$; Figure 2A and B). Remarkably, the serum ITGBL1 levels in early-stage HBV-HCC (41.66 [20.17-62.83] $\mathrm{ng} / \mathrm{mL}$ in the training cohort; 40.71 [22.79-57.57] $\mathrm{ng} / \mathrm{mL}$ in the validation cohort) were significantly lower compared to the at-risk group (Figure 2C and D).

We further carried out univariate and multivariate analyses to identify significant independent predictors of HBV$\mathrm{HCC}$ in these at-risk patients. Age, sex, $\mathrm{HBeAg}$, cirrhosis, albumin, AFP, and ITGBL1 were significantly correlated with HBV-HCC (Table 2). The multivariate results demonstrated lower ITGBL1 levels as an independent predictors factor for HBV-HCC occurring in these at-risk patients $(\mathrm{P}<0.0001$; Table 2). We also investigated whether cirrhosis and $\mathrm{HBeAg}$ level were influencing factors for the serum
ITGBL1 level in the HBV-HCC group. The results revealed that the level of serum ITGBL1 in the HBV-HCC group was independent of cirrhosis and $\mathrm{HBeAg}$. Furthermore, in the cirrhosis group, the serum level of ITGBL1 in HCC patients with cirrhosis was significantly lower than that in HBV-LC. Again, there was no significant difference in the serum levels of ITGBL1 between AFP-positive and AFP-negative patients in the HBV-HCC group, suggesting that regardless of the AFP level, the serum levels of ITGBL1 were significantly downregulated in the HBV-HCC group (Figure 3).

\section{Dynamic Changes of ITGBLI Serum} Levels in the Progression of Liver Disease To further investigate the dynamic changes in ITGBL1 serum levels in the progression of liver disease from 


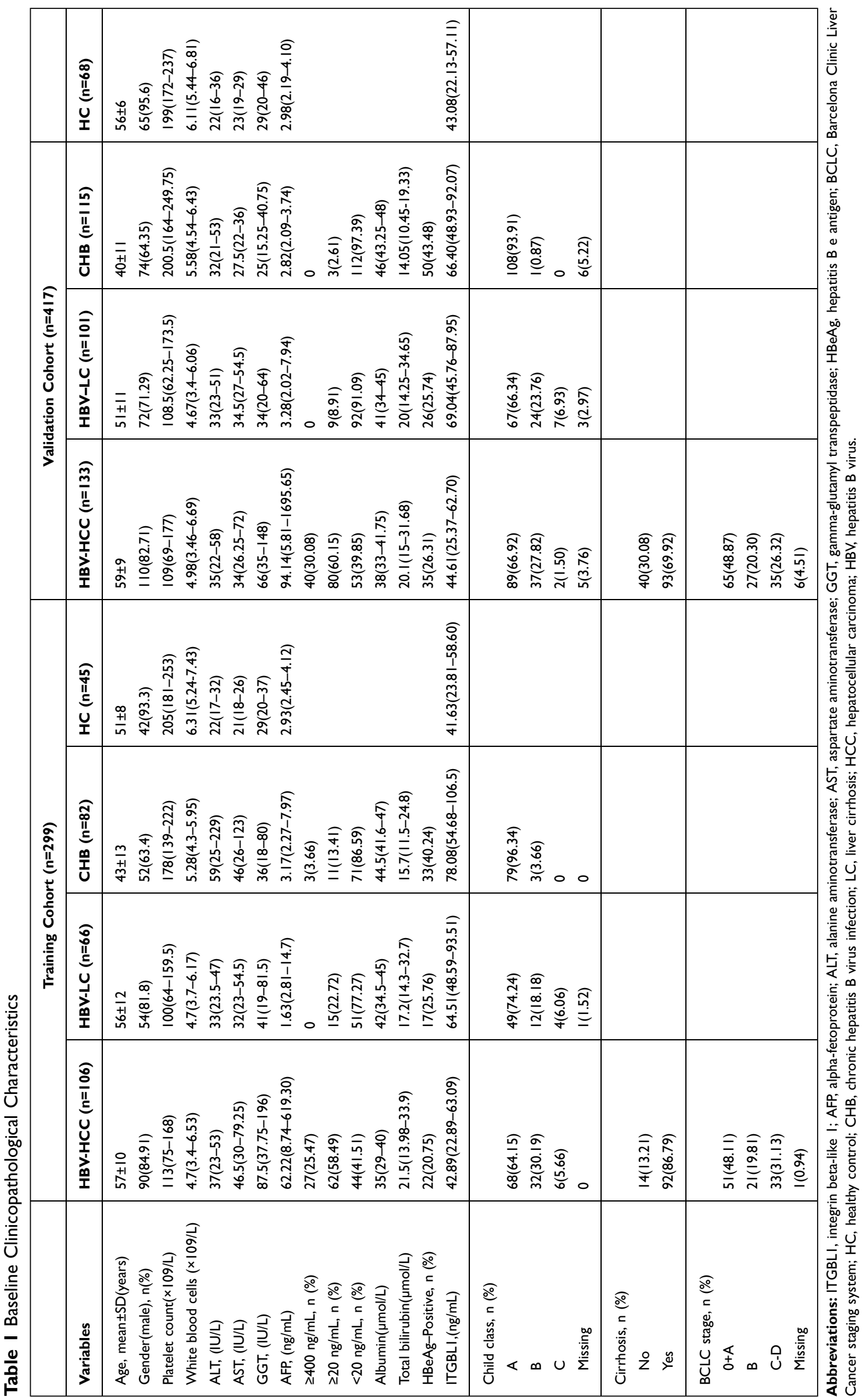


A

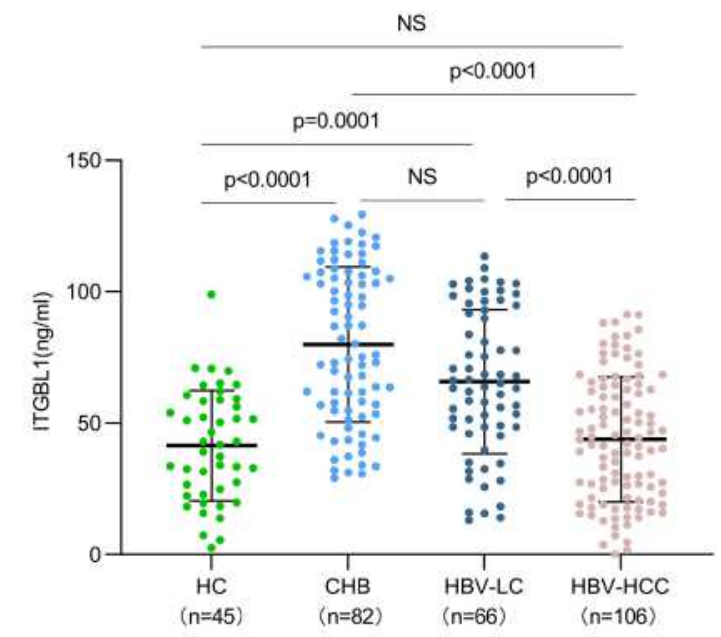

C

Training-Early-stage HCC

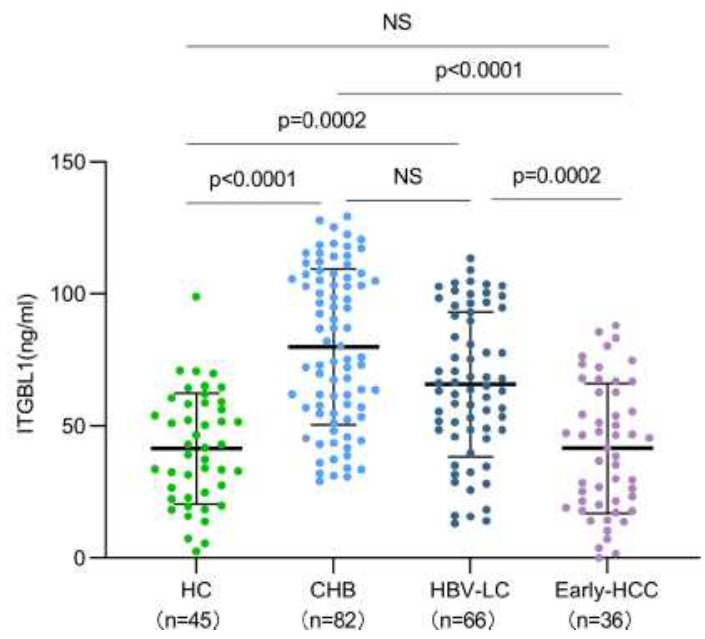

B

Validation-all HCC

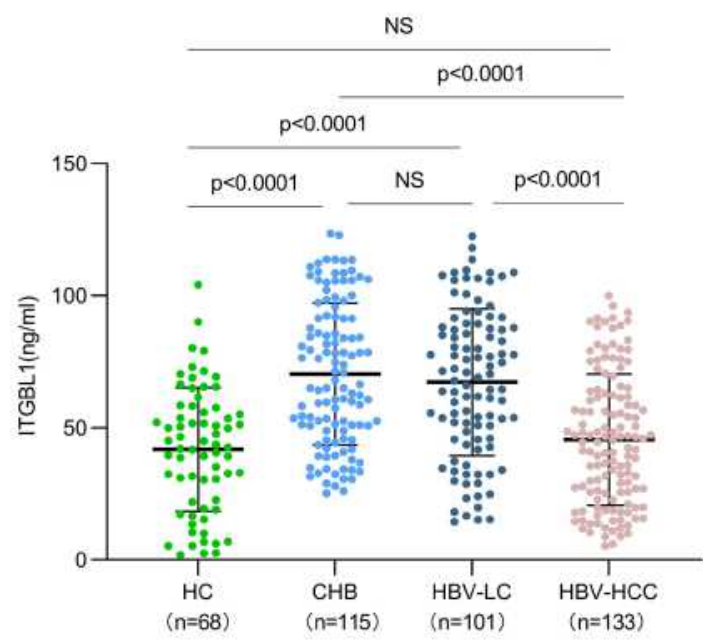

D

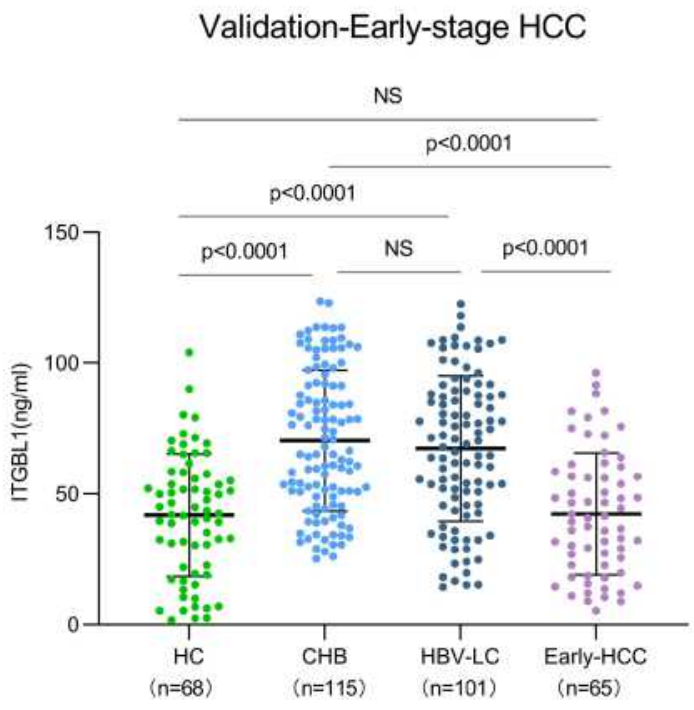

Figure 2 The level of ITGBLI in serum sample of the training and validation cohorts. The level of ITGBLI in all-HCC training cohort and validation cohort (A and B). The level of ITGBLI in early-stage HCC training cohort and validation cohort (C and D).

HBV-LC to HBV-HCC, we analysed the serum ITGBL1 levels of 41 patients who had progressed from HBV-LC to HBV-HCC during follow-up. Consistent with previous results, the serum ITGBL1 level in the HBV-HCC stage (41.49 [25.40-53.55] $\mathrm{ng} / \mathrm{mL}$ ) was significantly lower than that in the HBV-LC stage (74.87 [63.13-84.07] $\mathrm{ng} / \mathrm{mL}$, $\mathrm{P}<0.0001$; Figure 4A). Among those patients, series follow-up samples were obtained and tested from 19 patients before diagnosis: at 1 year, 3 years, 5 years and 10 years. The mean serum level of ITGBL1 in diagnosis time was
$27.10(20.52-48.12) \mathrm{ng} / \mathrm{mL}$ at 0 years, and values increased before diagnosis $(63.78$ [31.64-78.97] $\mathrm{ng} / \mathrm{mL}$ at 1 year, $83.69[68.25-107.8 . \mathrm{ng} / \mathrm{mL}$ at 3 years, 94.50 [72.50-104.2] $\mathrm{ng} / \mathrm{mL}$ at 5 years, and 83.83 [71.76-86.77] $\mathrm{ng} / \mathrm{mL}$ at 10 years). The results confirmed that ITGBL1 serum levels were downregulated once HBV-HCC developed from HBV-LC (Figure 4B and C). The level of downregulation of serum ITGBL1 was significantly changed, especially in patients with HBV-HCC within 1 year before diagnosis (Figure 4D). 


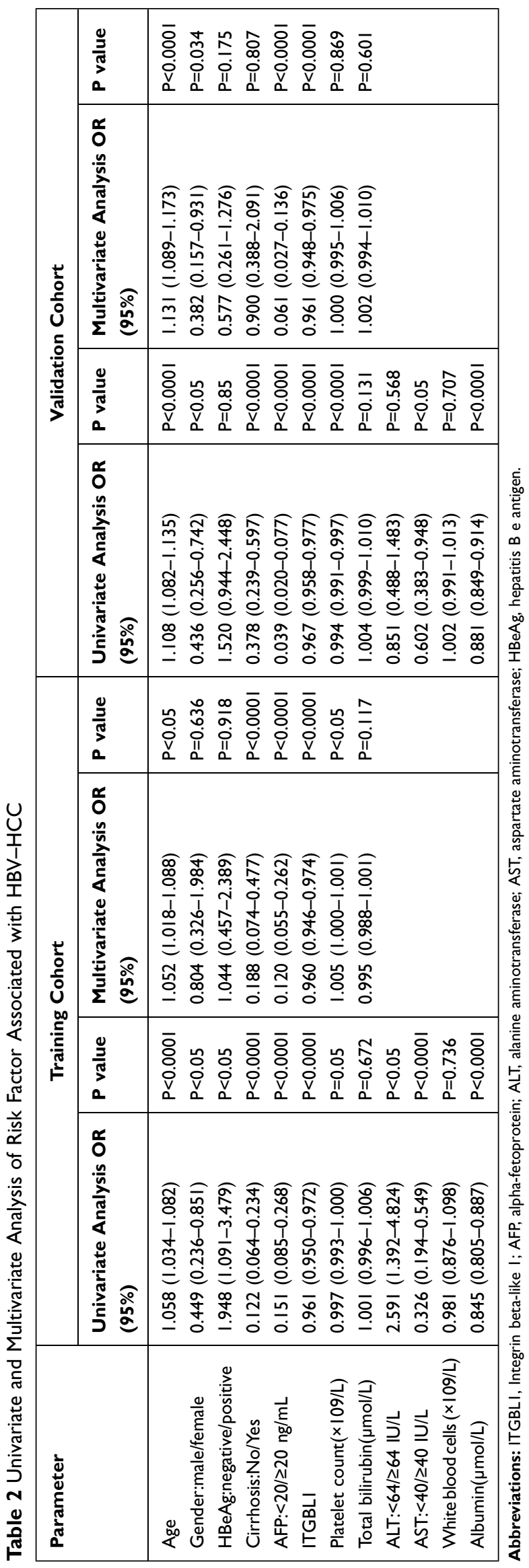

\section{Diagnostic Performance of the Serum Levels of ITGBLI \\ Diagnostic Performance for Serum ITGBLI for Early-Stage HBV-HCC}

In the training cohort, the optimal diagnostic cut-off value for ITGBL1 was $54.30 \mathrm{ng} / \mathrm{mL}$, and the AUC value for ITGBL1 was 0.773 (95\% confidence interval [CI], 0.716-0.823), with a sensitivity and specificity of $66.98 \%$ and $71.62 \%$, respectively, in distinguishing the HBV-HCC group from the at-risk group (Figure S1A, Table S2). Furthermore, 51 (48.11\%) of 106 patients with $\mathrm{HBV}-\mathrm{HCC}$ had an early-stage disease (BCLC stage 0+A) in the training cohort (Table 2). The diagnostic efficiency of ITGBL1 for distinguishing earlystage HBV-HCC from at-risk groups was evaluated and revealed an AUC of 0.787 (95\% CI, 0.723-0.842) and high specificity $(79.73 \%$; Figure 5A, Table 3) based on a diagnostic cut-off value $47.93 \mathrm{ng} / \mathrm{mL}$. Similar results were observed in the validation cohort that 65 (48.87\%) of 133 patients with HBV-HCC had an early-stage disease (BCLC stage $0+\mathrm{A}$ ) (Table 2 ). In conclusion, the serum ITGBL1 level is an effective biomarker for the diagnosis of early-stage $\mathrm{HBV}-\mathrm{HCC}$ from at-risk groups.

To compare the diagnostic accuracy of ITGBL1 with that of AFP, the most widely used HCC biomarker, we used 400 $\mathrm{ng} / \mathrm{mL}$ and $20 \mathrm{ng} / \mathrm{mL}$ (APF400 and APF20) as the recommended clinical cut-off values for AFP. For distinguishing $\mathrm{HBV}-\mathrm{HCC}$ patients from the at-risk group, the performance of ITGBL1 was significantly better than that of AFP400 $(\mathrm{P}<0.0001)$. When differentiating the HBV-HCC group from the HBV-LC group, the ITGBL1 level had an AUC of 0.721 (95\% CI, 0.648-0.787) and high specificity (77.27\%) in the training cohort (Figure S1C, Table S2). The performance of ITGBL1 was significantly better than that of AFP400 $(\mathrm{P}<0.05)$, and AFP20 have similar diagnostic efficiency $(p=0.44)$. For distinguishing the early-stage HBVHCC group from at-risk individuals, ITGBL1 (AUC, 0.787) had a better performance than AFP400 (AUC, 0.539; $<<0.0001$ ) and AFP20 (AUC, 0.638; $<<0.05$ ), while AFP400 and AFP20 identified early-stage HBV-HCC with higher specificity ( $97.97 \%$ and $82.43 \%$, respectively) in the training cohort (Figure 5A, Table 3). When only HBV-LC patients were included, the AUC for ITGBL1 in early-stage HBV-HCC was 0.738 (95\% CI, 0.648-0.815; sensitivity, $62.75 \%$; specificity, 77.27\%), whereas those of AFP400 and AFP20 were 0.549 and 0.612 (95\% CI, 0.454-0.641; sensitivity, 9.8\%; specificity, 100\%; and 95\% CI, $0.517-$ 0.701 ; sensitivity, $45.1 \%$; specificity, $77.27 \%$ ), respectively 

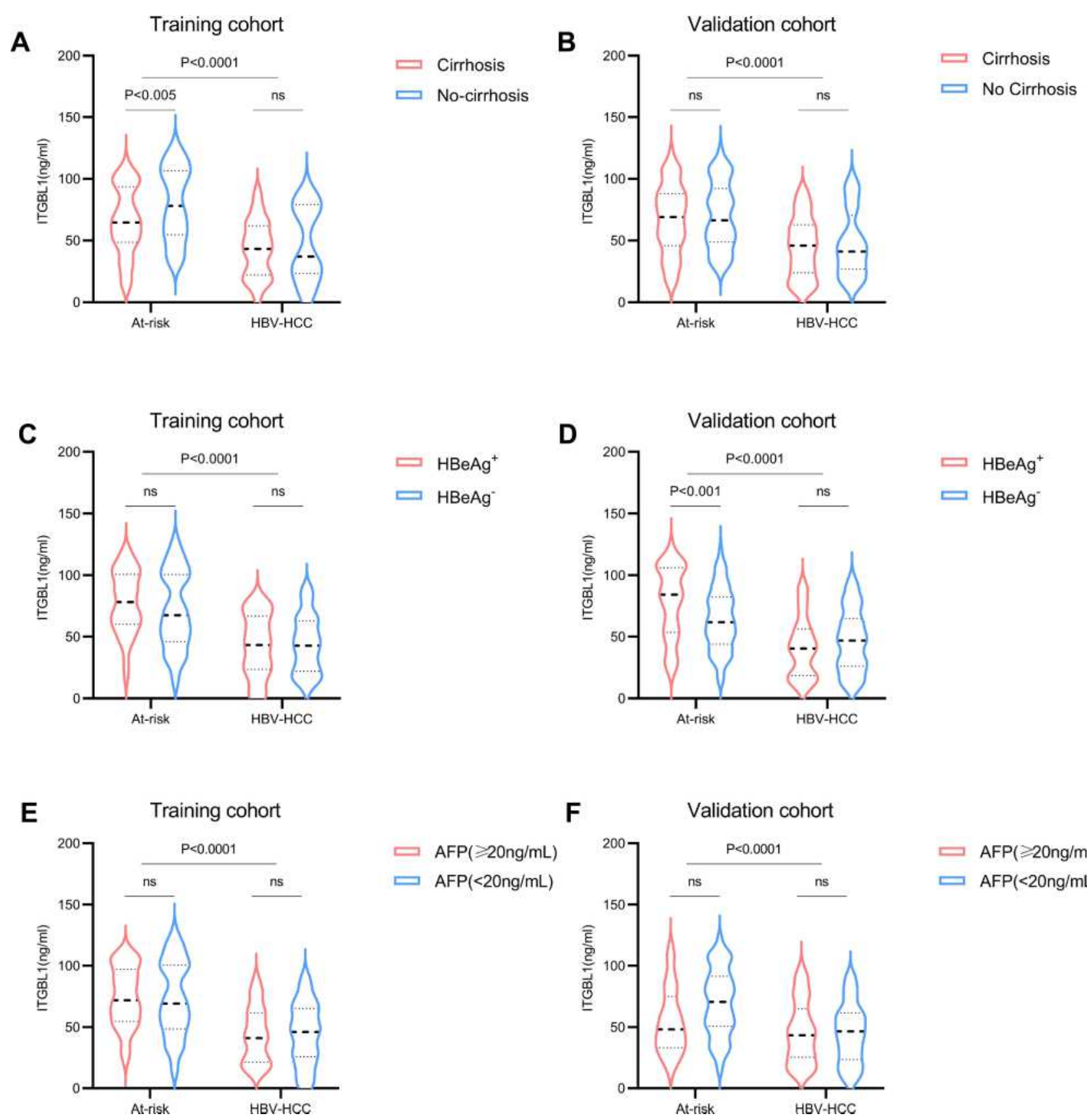

F Validation cohort

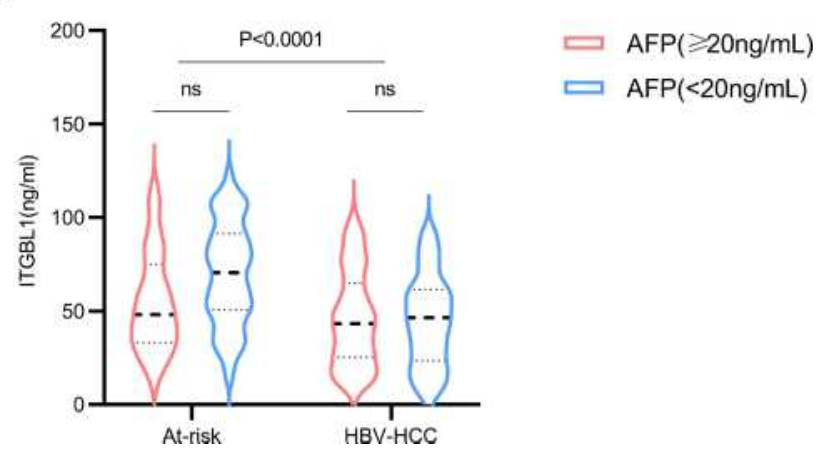

Figure 3 Comparison of ITGBLI levels in serum of At-risk and HBV-HCC patients in different subgroups. The level of ITGBLI in serum of the At-risk and HBV-HCC patients with or without cirrhosis in training and validation cohort $(\mathbf{A}$ and $\mathbf{B})$, with $\mathrm{HBeAg}^{+}$or $\mathrm{HBeAg}^{-}$in training and validation cohort $(\mathbf{C}$ and $\mathbf{D})$, with $\mathrm{AFP}<20 \mathrm{ng} / \mathrm{mL}$ or $A F P \geq 20 \mathrm{ng} / \mathrm{mL}$ in training and validation cohort ( $E$ and $\mathbf{F}$ ).

(Figure 5C, Table 3). The results in the validation cohort confirmed that ITGBL1 was better than AFP20 and AFP400 in diagnosing early-stage HBV-HCC in HBV-LC patients (Figure 5D, Table 3). In a word, the serum ITGBL1 level is more effective biomarker than AFP20 and AFP400 for the diagnosis of early-stage HBV-HCC.

The ROC analysis indicated that measurement of ITGBL1 combined with AFP20 significantly increased the diagnostic accuracy for HBV-HCC compared to either marker alone. ITGBL1 combined with AFP20 had the highest AUC with significantly higher specificity (AUC 0.840 [ $95 \%$ CI $0.789-0.883$ ], sensitivity $70.75 \%$, and specificity $82.43 \%$ in the training cohort; 0.868 [0.8280.902 ], $69.92 \%$, and $92.13 \%$ in the validation cohort) for distinguishing HBV-HCC patients from at-risk patients (Figure S1A-B, Table S2). When differentiating between HBV-HCC and HBV-LC patients, the performance of ITGBL1 combined with AFP20 had an AUC of 0.794 

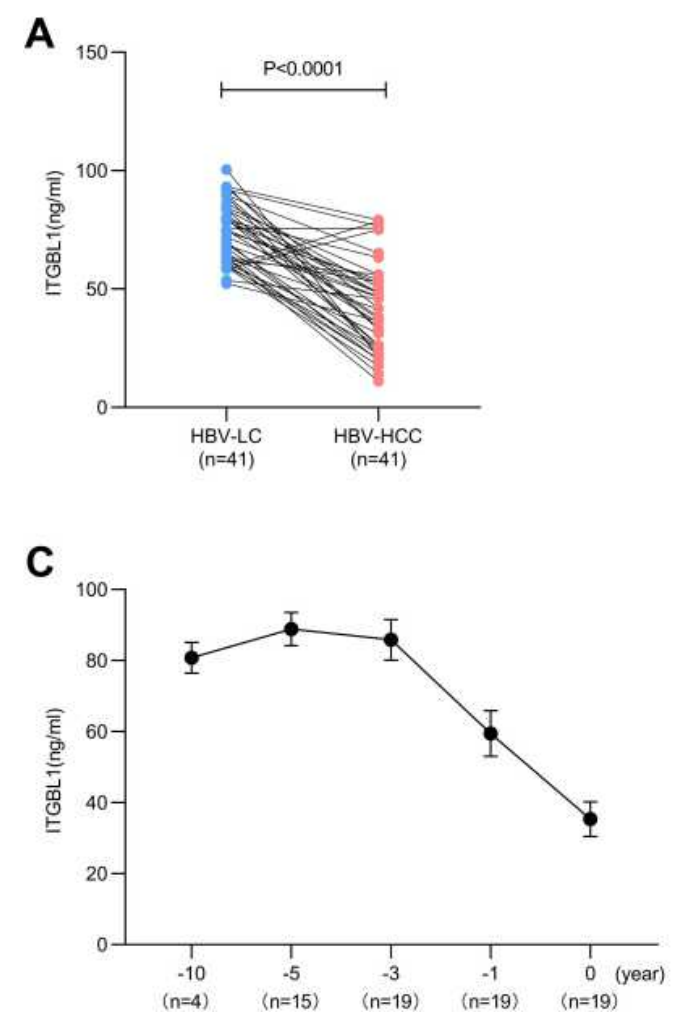

B
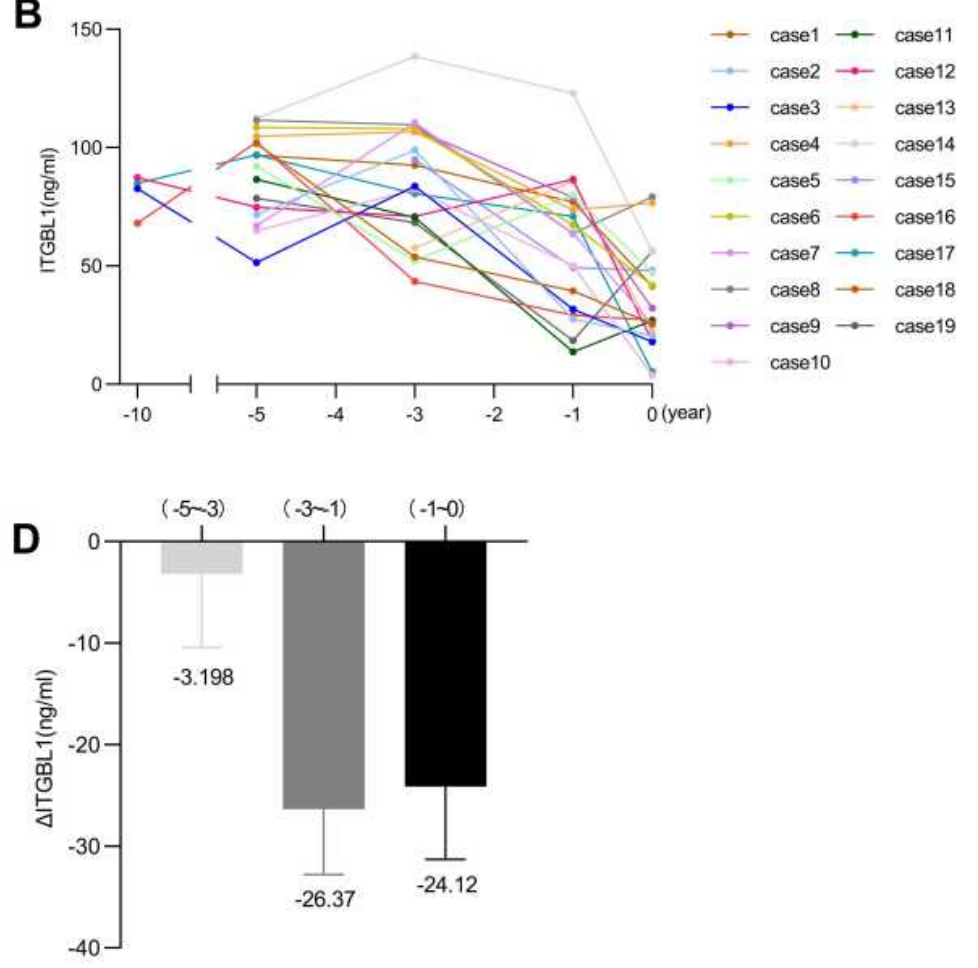

Figure 4 The dynamic change of ITGBLI level in serum of different patients. Comparison of ITGBLI levels in serum of 4 I patients from the HBV-LC to HBV-HCC (A). Kinetics of ITGBLI levels in serum in $19 \mathrm{HBV}-\mathrm{HCC}$ patients $(\mathbf{B})$. The mean level of ITGBLI in serum from diagnosis time $(0$ year) to before diagnosis $(-\mathrm{I},-3,-5$ and -10 years) (C). The change of $C$ in serum ITGBLI ( $\triangle I T G B L I)$ levels during pre-diagnostic follow-up (D).

(95\% CI, 0.726-0.852; sensitivity, 78.3\%; specificity, $68.18 \%)$ in the training cohort and 0.847 (95\% CI, 0.794-0.891; sensitivity, 64.66\%; specificity, 92.08\%) in the validation cohort (Figure S1C-D, Table S2). For differentiating patients with early-stage $\mathrm{HCC}$ from at-risk controls, the combination of ITGBL1 and AFP20 showed notably better performance than either test alone (AUC, 0.824 [95\% CI, 0.764-0.875]; sensitivity, 82.35\%; specificity, 68.24\%), and this was further confirmed in the validation group (AUC, 0.851 [95\% CI, 0.804-0.891]; sensitivity, 83.08\%; specificity, 71.30\%) (Figure 5A and $\mathrm{B}$, Table 3). When only HBV-LC controls were included, the AUC for ITGBL1 and AFP20 in the early-stage of HBV-HCC was 0.776 (95\% CI, 0.690-0.848; sensitivity, $76.47 \%$; specificity, 68.18\%) in the training cohort and 0.829 (95\% CI, 0.763-0.883; sensitivity, 83.08\%; specificity, 69.31\%) in the validation cohort (Figure 5C and D, Table 3).

We further explored the diagnostic accuracy of ITGBL1 for patients with early-stage HBV-HCC defined by different staging systems. The combination of ITGBL1 and AFP20 still improves the diagnostic accuracy of detecting early-stage HBV-HCC defined by different staging systems in the training and validation cohorts (Table S3).

\section{Diagnostic Performance for Serum ITGBLI for AFP-Negative HBV-HCC and Early-Stage HBV-HCC Patients}

In addition, we investigated the diagnostic effectiveness of ITGBL1 in $\mathrm{AFP}<400 \mathrm{ng} / \mathrm{mL}$ and AFP-negative $(\mathrm{AFP}<20$ $\mathrm{ng} / \mathrm{mL}$ ) $\mathrm{HCC}$ patients. In the training and validation cohorts, $\mathrm{HBV}-\mathrm{HCC}$ patients with $\mathrm{AFP}<400 \mathrm{ng} / \mathrm{mL}$ accounted for $74.53 \%$ (79 of 106) and $69.92 \%$ (93 of 133) of the participants, respectively (Table 2). The AUC of serum ITGBL1 was 0.772 (95\% CI, 0.712-0.825; sensitivity, 70.89\%; specificity, 69.66\%) in the training cohort and 0.745 (95\% CI, 0.693-0.793; sensitivity, 65.59\%; specificity, 74.07\%) in the validation cohort for distinguishing $\mathrm{AFP}<400 \mathrm{ng} / \mathrm{mL} \mathrm{HBV}-$ HCC patients from at-risk controls (Figure S2A-B, Table S4). When only HBV-LC controls were included, the AUC for ITGBL1 in the $\mathrm{AFP}<400 \mathrm{ng} / \mathrm{mL}$ group of $\mathrm{HBV}-\mathrm{HCC}$ patients was 0.723 (95\% CI, 0.642-0.794; sensitivity, $59.49 \%$; specificity, $77.27 \%$ ) in the training cohort and 0.730 (95\% CI, 0.662-0.791; sensitivity, 65.59\%; specificity, $73.17 \%$ ) in the validation cohort (Figure S2C-D, Table 
A

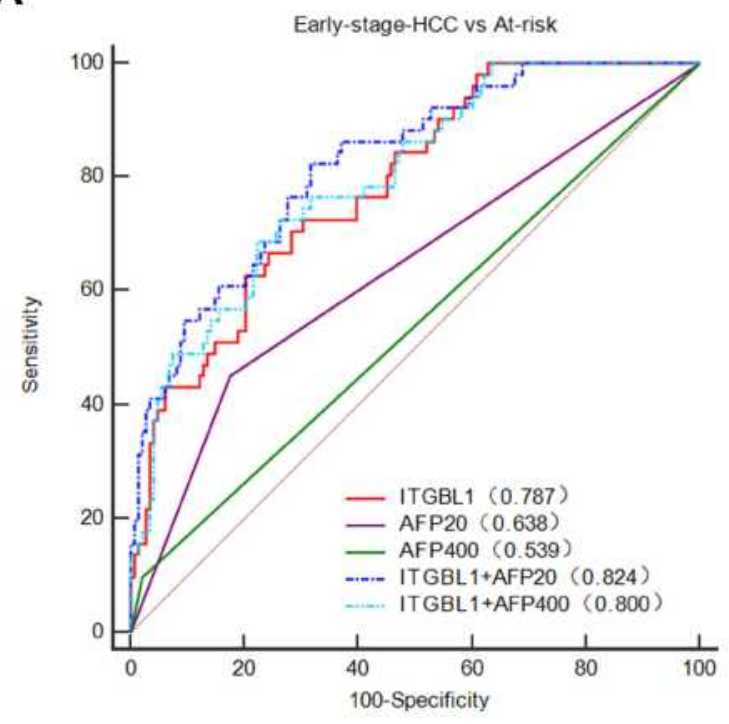

C

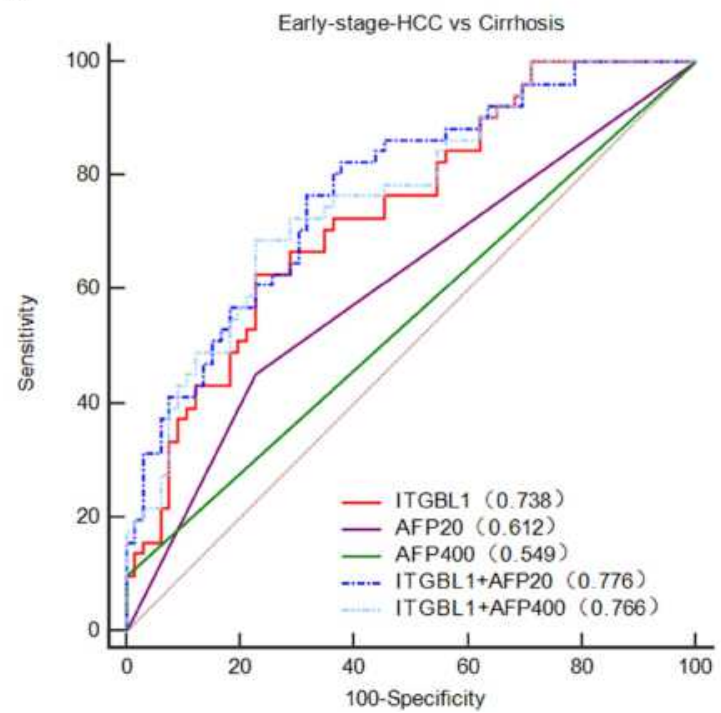

B

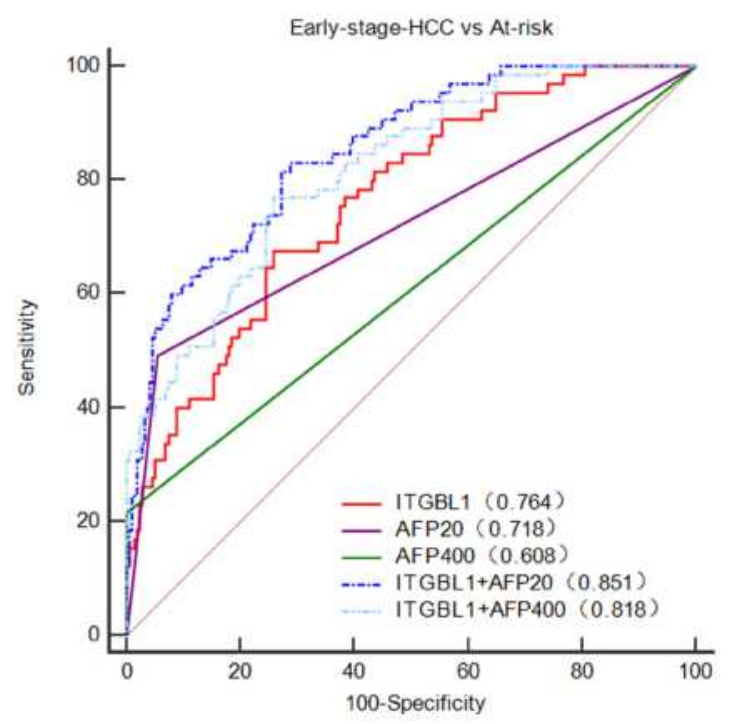

D

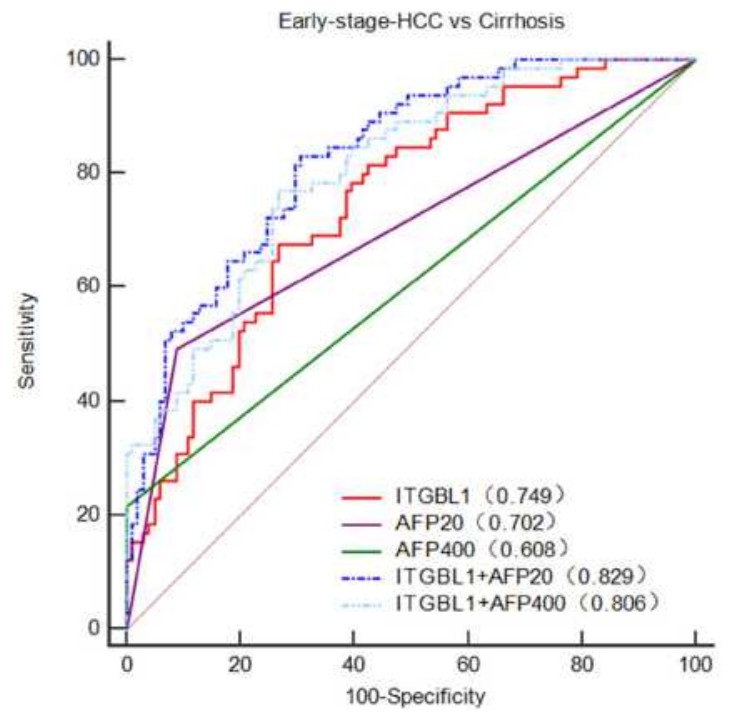

Figure 5 Diagnostic performance for serum ITGBLI for early-stage HBV-HCC. ROC curve for ITGBLI, AFP, or both, for patients with early-stage HBV-HCC versus controls at risk of HBV-HCC in the training and validation cohort (A and B). ROC curve for ITGBLI, AFP, or both, for patients with the early-stage HBV-HCC versus controls at HBV-LC in the training and validation cohort (C and D).

S4). The AFP-negative (AFP $<20 \quad \mathrm{ng} / \mathrm{mL})$ HBV-HCC patients accounted for 44 of 106 (41.51\%) participants in the training cohort and 53 of 133 (39.85\%) patients in the validation cohort (Table 2). When AFP-negative at-risk control individuals were included, ITGBL1 also showed a better performance in the AFP-negative HBV-HCC group (AUC 0.756 [95\% CI 0.683-0.819], sensitivity $68.18 \%$, and specificity $68.85 \%$ in the training cohort; 0.744 [0.686-0.796], $81.13 \%$, and $55.88 \%$ in the validation cohort; Figure 6A and B, Table S4). For distinguishing
AFP-negative HBV-HCC patients from HBV-LC controls, the AUC of serum ITGBL1 was 0.698 (95\% CI, 0.5950.788 ; sensitivity, $61.39 \%$; specificity, $70.59 \%$ ) in the training cohort and 0.737 (95\% CI, 0.658-0.807; sensitivity, $79.25 \%$; specificity, 59.78\%) in the validation cohort (Figure 6C and D, Table S4).

For patients with early-stage HBV-HCC from the atrisk group, 46 of $51(90.20 \%) \mathrm{AFP}<400 \mathrm{ng} / \mathrm{mL}$ patients in the training stage and 51 of $65(78.46 \%) \mathrm{AFP}<400 \mathrm{ng} /$ $\mathrm{mL}$ patients in the validation stage, the AUC of serum 
Table 3 The Value of AFP and ITGBLI Alone or in Combination in the Diagnosis of the Early-Stage HBV-Related HCC

\begin{tabular}{|c|c|c|c|c|c|c|c|}
\hline & AUC $(95 \% \mathrm{Cl})$ & Sensitivity(\%) & Specificity(\%) & PPV(\%) & NPV(\%) & Positive LR & Negative LR \\
\hline \multicolumn{8}{|c|}{ Early-stage HCC vs At-risk } \\
\hline \multicolumn{8}{|l|}{ Training } \\
\hline ITGBLI & $0.787(0.723-0.842)$ & 62.75 & 79.73 & 51.6 & 86.1 & 3.1 & 0.47 \\
\hline AFP20 & $0.638(0.567-0.704)$ & 45.10 & 82.43 & 46.9 & 81.3 & 2.57 & 0.67 \\
\hline AFP400 & $0.539(0.467-0.610)$ & 9.80 & 97.97 & 62.5 & 75.9 & 4.84 & 0.92 \\
\hline ITGBLI+AFP20 & $0.824(0.764-0.875)$ & 82.35 & 68.24 & 47.2 & 91.8 & 2.59 & 0.26 \\
\hline ITGBLI+AFP400 & $0.800(0.737-0.853)$ & 68.63 & 77.70 & 51.5 & 87.8 & 3.08 & 0.40 \\
\hline \multicolumn{8}{|l|}{ Validation } \\
\hline ITGBLI & $0.764(0.7 \mid 0-0.813)$ & 67.69 & 74.07 & 44.0 & 88.4 & 2.61 & 0.44 \\
\hline AFP20 & $0.718(0.662-0.770)$ & 49.23 & 94.44 & 72.7 & 86.1 & 8.86 & 0.54 \\
\hline AFP400 & $0.608(0.548-0.665)$ & 21.54 & 100 & 100 & 80.9 & & 0.78 \\
\hline ITGBLI+AFP20 & $0.85 I(0.804-0.891)$ & 83.08 & 71.30 & 46.6 & 93.3 & 2.89 & 0.24 \\
\hline ITGBLI+AFP400 & $0.818(0.767-0.861)$ & 76.92 & 74.07 & 47.2 & 91.4 & 2.97 & 0.31 \\
\hline \multicolumn{8}{|c|}{ Early-stage HCC vs LC } \\
\hline \multicolumn{8}{|l|}{ Training } \\
\hline ITGBLI & $0.738(0.648-0.815)$ & 62.75 & 77.27 & 68.1 & 72.9 & 2.76 & 0.48 \\
\hline AFP20 & $0.612(0.517-0.701)$ & 45.10 & 77.27 & 60.5 & 64.6 & 1.98 & 0.71 \\
\hline AFP400 & $0.549(0.454-0.64 I)$ & 9.80 & 100 & 100 & 58.9 & & 0.9 \\
\hline ITGBLI+AFP20 & $0.776(0.690-0.848)$ & 76.47 & 68.18 & 65.0 & 78.9 & 2.4 & 0.35 \\
\hline ITGBLI+AFP400 & $0.766(0.679-0.839)$ & 68.63 & 77.27 & 70.0 & 76.1 & 3.02 & $0.4 I$ \\
\hline \multicolumn{8}{|l|}{ Validation } \\
\hline ITGBLI & $0.749(0.676-0.813)$ & 67.69 & 73.27 & 62.0 & 77.9 & 2.53 & 0.44 \\
\hline AFP20 & $0.702(0.626-0.770)$ & 49.23 & 91.09 & 78.0 & 73.6 & 5.52 & 0.56 \\
\hline AFP400 & $0.608(0.529-0.682)$ & 21.54 & 100 & 100 & 66.4 & & 0.78 \\
\hline ITGBLI+AFP20 & $0.829(0.763-0.883)$ & 83.08 & 69.31 & 63.5 & 86.4 & 2.71 & 0.24 \\
\hline ITGBLI+AFP400 & $0.806(0.737-0.863)$ & 76.92 & 73.27 & 64.9 & 83.1 & 2.88 & 0.31 \\
\hline
\end{tabular}

Abbreviations: ITGBLI, integrin beta-like I; AFP, $\alpha$-fetoprotein; AUC, area under curve; $95 \% \mathrm{Cl}, 95 \%$ confidence interval; PPV, positive predictive value; NPV, negative predictive value; LR, likelihood ratio; HCC, hepatocellular carcinoma; LC, liver cirrhosis; at-risk controls, patients with chronic hepatitis B and HBV-related cirrhosis; AFP20, $20 \mathrm{ng} / \mathrm{mL}$ of alpha fetoprotein as a cutoff value; AFP400, $400 \mathrm{ng} / \mathrm{mL}$ of alpha fetoprotein as a cutoff value; AUC, area under curve.

ITGBL1 was $0.788(95 \% \mathrm{CI}, 0.723-0.844$; sensitivity, $69.57 \%$; specificity, $75.17 \%$ ) in the training cohort and 0.768 (95\% CI, 0.712-0.817; sensitivity, 70.59\%; specificity, $74.07 \%$ ) in the validation cohort (Figure S2E-F, Table S4). When only HBV-LC controls were included, the AUC for ITGBL1 in early-stage HBV-HCC patients with $\mathrm{AFP}<400 \mathrm{ng} / \mathrm{mL}$ was 0.740 (95\% CI, 0.649-0.819; sensitivity, $65.22 \%$; specificity, $77.27 \%$ ) in the training cohort and 0.752 (95\% CI, 0.676-0.819; sensitivity, $70.59 \%$; specificity, $73.27 \%$ ) in the validation cohort (Figure S2G-H, Table S4). Interestingly, ITGBL1 was also valuable to assess AFP-negative patients with earlystage HCC. In 28 of $51(54.90 \%)$ patients in the training cohort and 33 of $65(50.77 \%)$ patients in the validation cohort, serum ITGBL1 concentrations could distinguish patients with early-stage $\mathrm{HCC}$ from the at-risk group. The AUC of serum ITGBL1 for AFP-negative earlystage HBV-HCC patients from the at-risk group was 0.786 (95\% CI, 0.712-0.849; sensitivity, 75.0\%; specificity, 68.85\%) in the training cohort and $0.767(95 \% \mathrm{CI}$, $0.708-0.819$; sensitivity, 66.67\%; specificity, $75.49 \%$ ) in the validation cohort (Figure 6E and F, Table S4). When only HBV-LC controls were included, the AUC for ITGBL1 in early-stage AFP-negative HBV-HCC was 0.733 (95\% CI, 0.622-0.826; sensitivity, 67.86\%; specificity, $70.59 \%)$ in the training cohort and $0.764(95 \% \mathrm{CI}$, 0.680-0.835; sensitivity, $87.88 \%$; specificity, $55.43 \%$ ) in the validation cohort (Figure 6G and $\mathrm{H}$, Table S4). 
A

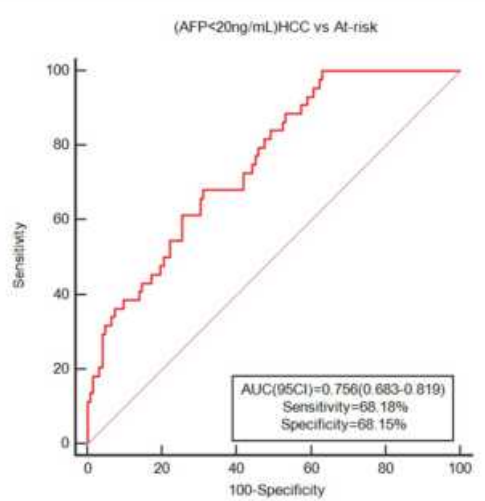

C

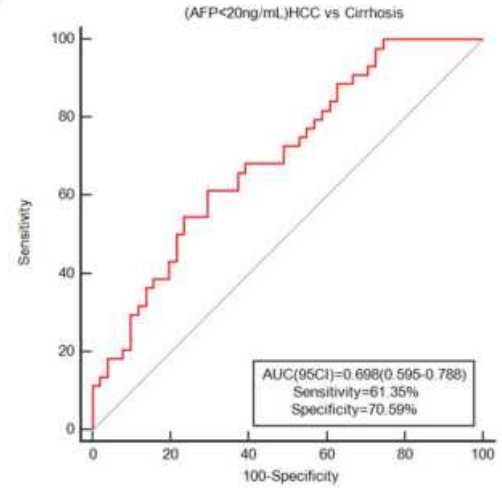

E

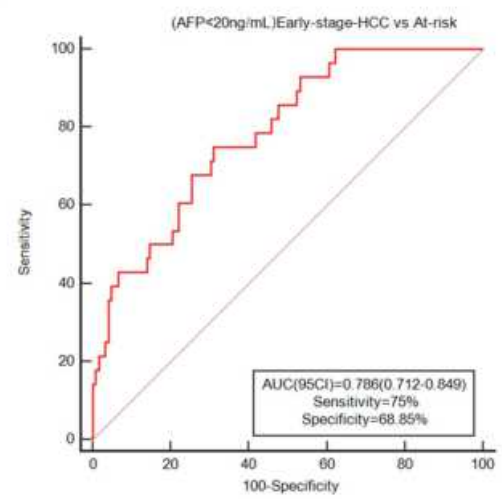

G

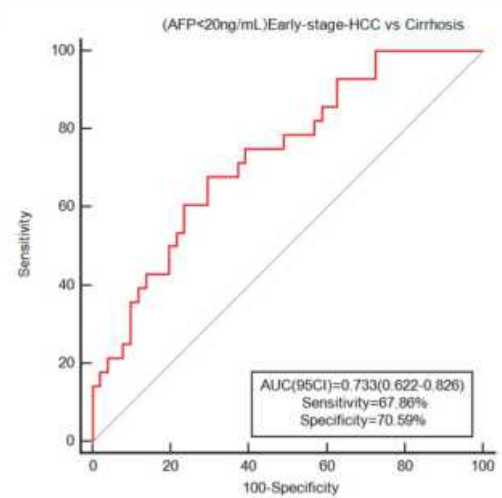

B

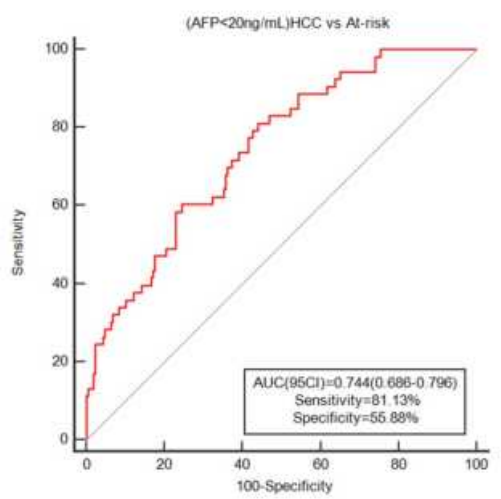

D

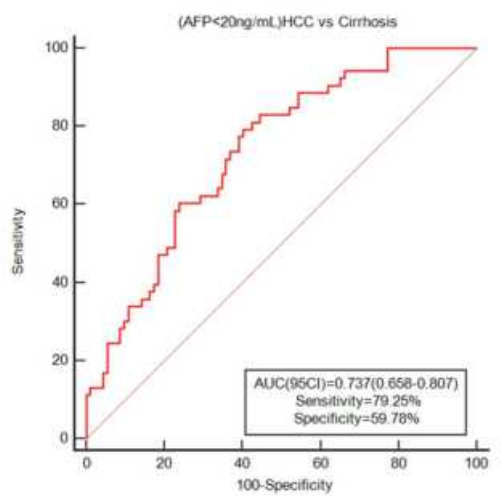

F

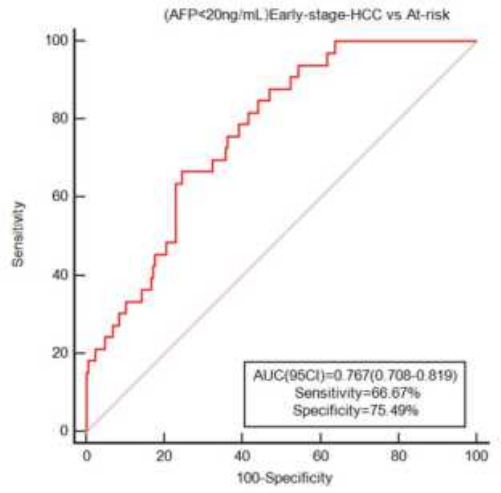

H

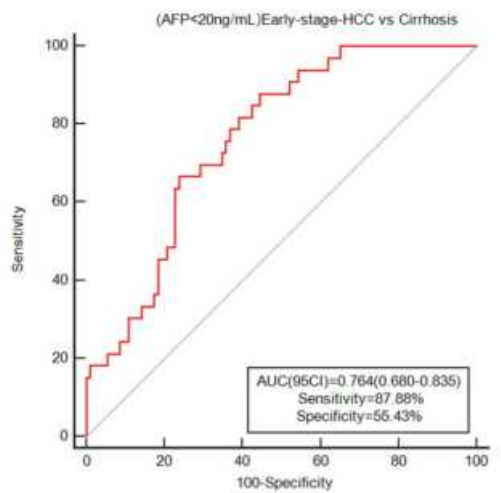

Figure 6 Diagnostic performance for serum ITGBLI for AFP-negative HBV-HCC and early-stage HBV-HCC patients. ROC curve for ITGBLI distinguishing individuals with AFP-negative HBV-HCC from at-risk groups in the training and validation cohort (A and B), from HBV-LC patients in the training and validation cohort (C and $\mathbf{D})$. ROC curve of ITGBLI distinguishing individuals with AFP-negative early-stage HBV-HCC from at-risk groups the training and validation cohort (E and F). ROC curve of ITGBLI distinguishing individuals with AFP-negative $\mathrm{HBV}-\mathrm{HCC}$ from $\mathrm{HBV}-\mathrm{HCC}$ patients the training and validation cohort $(\mathbf{G}$ and $\mathbf{H})$. 
Our results indicated that serum ITGBL1 was a valuable serum biomarker for diagnosing HBV-HCC in the early stage, and it is not correlated to the AFP status.

\section{Discussion}

In this study, we analysed serum ITGBL1 concentrations in training and validation cohorts of HBV-HCC patients and control subjects. Based on our results, the measurement of serum ITGBL1 has a promising diagnostic value for HBV-HCC, especially in AFP-negative (AFP $<20 \mathrm{ng}$ / $\mathrm{mL}$ ) patients and patients with early-stage HBV-HCC from at-risk populations. Furthermore, the combination of ITGBL1 and AFP20 has a higher efficiency than the use of a single biomarker in the early diagnosis of liver cancer.

Various studies have implicated ITGBL1 in tumour progression. ITGBL1 promotes bone metastasis of breast cancer cells through the TGF- $\beta$ signalling pathway. ${ }^{16}$ In ovarian cancer ${ }^{15}$ and gastrointestinal cancer, ${ }^{17-20}$ ITGBL1 was significantly upregulated and identified as a key regulator of tumour cell metastasis and invasion. In non-small cell lung cancer, downregulated ITGBL1 was frequently observed to inhibit cancer cell migration and invasion through the Wingless-Type MMTV Integration Site Family (Wnt)/ Planar cell polarity(PCP) signalling pathway. ${ }^{30}$ Previous studies demonstrated that in CHB patients with liver fibrosis and HCC, ITGBL1 expression level in liver tissue is upregulated and plays an important role in the progression of liver disease. ${ }^{21-23}$

In the present study, one unexpected result was that the serum ITGBL1 level in the HBV-HCC group was significantly lower than that among CHB and HBV-LC patients, which is contrary to previous results from tissue studies. ${ }^{23}$ To confirm our results, besides the incorporation of more patients from the other two hospitals, we further tested series follow-up samples from patients who progressed from HBV-LC to HCC. As shown in Figure 4, the dynamic change in ITGBL1 serum level provided convincing evidence that ITGBL1 serum levels decreased significantly during progression to HCC in most follow-up patients. The reason for this discrepancy is currently unclear. One possible explanation is that ITGBL1 may be released by HCC tissues into the serum in a specific secretory manner, such as via exosomes. One study demonstrated that in a colorectal cancer model, high ITGBL1rich extracellular vesicles could facilitate colorectal cancer metastatic growth in vivo. ${ }^{31}$ Another possible mechanism may correlated with protein-deficient secretion. These hypotheses need further exploration. Notably, in our previous study on ITGBL1 expression and liver fibrosis stage, ITGBL1 expression levels in liver tissues were upregulated in fibrosis patients compared to CHB patients, while no significant difference in serum levels was observed between the two groups (data not published), which is similar to our results in CHB and HBV-LC patients. $^{22}$ Our findings also revealed that the low serum ITGBL1 levels in the HBV-HCC group remained "stable," ie independent of cirrhosis and $\mathrm{HBeAg}$ status. These results imply that ITGBL1 might play a complex role in the three main stages of liver disease progression from CHB to HBV-HCC.

Another interesting result was that the serum level of ITGBL1 in healthy control (HC) is almost similar with that in HBV-HCC, which meant it is difficult to directly distinguish HCC from HC just based on the ITGBL1 level. However, firstly, in HBV infected (HBsAg positive) population, as shown above, ITGBL1 levels showed a dynamic change from $\mathrm{CHB}$ and HBV-LC to HBV-HCC, and were significant different between non-HCC (CHB and HBVLC) and HBV-HCC including early stage of HBV-HCC. Secondly, as shown in Figure 3 and Figure S3-5, the ITGBL1 levels were independent of HBeAg, HBV DNA, ALT, AFP levels and antiviral therapy or not. Given HBV infection remained a major contributor to HCC especially in medium and high endemic-area, monitoring of serum ITGBL1 level or its dynamic change could play a valuable role in differentiating the $\mathrm{HBV}-\mathrm{HCC}$ from $\mathrm{HBsAg}$ positive patients.

AFP is still the most widely accepted serum biomarker used in HCC diagnosis, but its performance is suboptimal because $30-40 \%$ of HCC patients are AFP-negative, according to some reports. ${ }^{32}$ Indeed, in our study, AFPnegative HCC patients comprised $41.51 \%$ and $39.85 \%$ of the training and validation cohorts, respectively; notably, AFP-negative early-stage HCC patients were $54.90 \%$ of the patients in the training cohort and $50.77 \%$ in the validation cohort (Table 2). Some potential biomarkers, such as AFP complementary methods, have been developed in the last two decades to increase the diagnostic accuracy of AFP-negative or early-stage HCC. In our study, we also observed no significant difference in ITGBL1 levels between AFP-positive and AFP-negative patients in the HBV-HCC group. The ROC analysis confirmed that ITGBL1 was still superior to $\mathrm{AFP}<400 \mathrm{ng} / \mathrm{mL}$ and $\mathrm{AFP}<20 \mathrm{ng} / \mathrm{mL}$ in the diagnosis of HBV-HCC, especially for early HBV-HCC in AFP-negative patients (Figure 6). 
Based on univariate and multivariate analyses in the present study, the serum ITGBL1 level was a significant independent predictor of HBV-HCC in these at-risk patients, and the dynamic changes in ITGBL1 serum levels in the progression of liver disease from HBV-LC to HBV-HCC suggested the feasibility of serum ITGBL1 in diagnosing HBV-HCC before clinical diagnosis. For all HBV-HCC patients, the serum ITGBL1 had a better AUC value of 0.773 and sensitivity of $66.98 \%$ compared to AFP400 and AFP20 in detecting liver cancer in patients with CHB and HBV-LC. When combined with AFP20, the AUC was largest at 0.840 , with $70.75 \%$ sensitivity and $82.43 \%$ sensitivity (Figure S1, Table S2). The detection and screening of early-stage HBV-HCC are associated with improved outcomes in patients. ${ }^{5}$ In this study, ITGBL1 also showed better diagnostic performance (AUC, 0.787; sensitivity, 62.75\%) than AFP400 and AFP20 in at-risk populations. Furthermore, we evaluated the diagnostic accuracy of ITGBL1 for patients with earlystage HBV-HCC according to different definitions of early tumour occurrence. The combination of ITGBL1 and AFP20 still improves the diagnostic accuracy of detecting early-stage HBV-HCC defined by different staging systems in the training and validation cohorts (Figure 5, Table 3).

The incidence of HCC in patients with HBV-related liver cirrhosis is $3-8 \%$ per year, and these patients are considered an "extremely high-risk group" by the Japan Society of Hepatology guidelines. ${ }^{33}$ Because of the increased liver nodularity and parenchymal heterogeneity of liver cirrhosis, the sensitivity of ultrasound examination is limited, and AFP is usually used as a supplemental test. As shown in Table 3, the diagnostic performance (AUC) of ITGBL1 for distinguishing HBV-HCC from HBV-LC was comparable to that of AFP20, as for distinguishing early-stage HCC from HBV-LC, the AUC of ITGBL1 was greater than that of AFP20. Both diagnostic accuracies reached the highest value when combining ITGBL1 and AFP20. Of note, as shown in Figure $4 \mathrm{C}$ and D, serum ITGBL1 levels were significantly downregulated at 3 years before HCC diagnosis, which might be an early molecular event of liver cirrhosis progressing to HCC. However, this conclusion needs to be confirmed by further experiments.

We acknowledge that there are a few limitations to this study. First, HCC is a heterogeneous disease with multiple underlying aetiologies, but this study population was focused on HCC patients mostly with $\mathrm{CHB} / \mathrm{LC}$ background in China. Therefore, the results should be interpreted carefully for patient populations with different aetiological backgrounds, and further studies are needed to evaluate the performance of the ITGBL1 in diagnosing HCC patients with a variety of liver diseases originating from different geographical regions. Second, although major clinical variables were controlled in our analyses, selection bias was unavoidable in the cross-sectional and retrospective study. Large-scale prospective studies are needed to further confirm and establish the ultimate clinical utility of this approach for HCC. Third, our study only showed that serum levels of ITGBL1 declined significantly in patients with early-stage HBV-HCC compared to CHB and HBV-LC controls. We did not explain the inconsistency between ITGBL1 levels in serum and tissue, and the molecular mechanism and clinical value of this difference also need to be further explored. Considering that little is known about ITGBL1 and its role in the pathogenesis of HBV-HCC, this area warrants further research. Therefore, we consider further functional studies that will provide insights into the potential mechanisms of ITGBL1 detected in HBV-HCC.

In summary, our results provide strong evidence that serum ITGBL1 could serve as a non-invasive and effective diagnostic biomarker for the early diagnosis of HBV-HCC in at-risk patients. The combination of ITGBL1 and AFP20 could notably increase the diagnostic performance and might provide a new strategy for the diagnosis of early-stage $\mathrm{HCC}$ in these patients. Moreover, the measurement of ITGBL1 in serum could complement AFP in the diagnosis of HBV-HCC and resolve the deficiencies of AFP in the testing of AFP-negative patients to distinguish HBV-HCC from non-malignant chronic liver diseases.

\section{Conclusion}

The serum level of ITGBL1 improved identification of AFP-negative HBV-HCC patients, and increased diagnostic accuracy with AFP20 together in the early detection of HBV-HCC.

\section{Funding}

This study was financially supported by the grants from the National Science and Technology Major Project of China (2018ZX10302205), National Natural Science Foundation of China (81572047) and Chinese foundation for hepatitis prevention and control-TianQing liver disease research fund subject (TQGB20210029), Natural Science 
Foundation of Shanghai (20ZR1434100), Shanghai Science and Technology Commission (19411971500).

\section{Disclosure}

The authors reports no conflicts of interest in this work.

\section{References}

1. Bray F, Ferlay J, Soerjomataram I, Siegel RL, Torre LA, Jemal A. Global cancer statistics 2018: GLOBOCAN estimates of incidence and mortality worldwide for 36 cancers in 185 countries. CA Cancer J Clin. 2018;68(6):394-424. doi:10.3322/caac.21492

2. Villanueva A. Hepatocellular Carcinoma. N Engl J Med. 2019;380 (15):1450-1462. doi:10.1056/NEJMra1713263

3. Wong MCS, Huang JLW, George J, et al. The changing epidemiology of liver diseases in the Asia-Pacific region. Nat Rev Gastroenterol Hepatol. 2019;16(1):57-73. doi:10.1038/s41575-018-0055-0

4. Chen W, Zheng R, Baade PD, et al. Cancer statistics in China, 2015. CA Cancer J Clin. 2016;66(2):115-132. doi:10.3322/caac.21338

5. Yang JD, Hainaut P, Gores GJ, Amadou A, Plymoth A, Roberts LR. A global view of hepatocellular carcinoma: trends, risk, prevention and management. Nat Rev Gastroenterol Hepatol. 2019;16 (10):589-604. doi:10.1038/s41575-019-0186-y

6. Sapisochin G, Bruix J. Liver transplantation for hepatocellular carcinoma: outcomes and novel surgical approaches. Nat Rev Gastroenterol Hepatol. 2017;14(4):203-217. doi:10.1038/nrgastro. 2016.193

7. Forner A, Reig M, Bruix J. Hepatocellular carcinoma. The Lancet. 2018;391(10127):1301-1314. doi:10.1016/s0140-6736(18)30010-2

8. Heimbach JK, Kulik LM, Finn RS, et al. AASLD guidelines for the treatment of hepatocellular carcinoma. Hepatology. 2018;67 (1):358-380. doi:10.1002/hep.29086

9. Singal AG, Conjeevaram HS, Volk ML, et al. Effectiveness of hepatocellular carcinoma surveillance in patients with cirrhosis. Cancer Epidemiol Biomarkers Prev. 2012;21(5):793-799. doi:10.1158/10559965.EPI-11-1005

10. Simmons O, Fetzer DT, Yokoo T, et al. Predictors of adequate ultrasound quality for hepatocellular carcinoma surveillance in patients with cirrhosis. Aliment Pharmacol Ther. 2017;45 (1):169-177. doi:10.1111/apt.13841

11. Quaglia A. Hepatocellular carcinoma: a review of diagnostic challenges for the pathologist. J Hepatocell Carcinoma. 2018;5:99-108. doi:10.2147/JHC.S159808

12. Kim MN, Kim BK, Kim SU, et al. Longitudinal assessment of alpha-fetoprotein for early detection of hepatocellular carcinoma in patients with cirrhosis. Scand J Gastroenterol. 2019;54(10): 1283-1290. doi:10.1080/00365521.2019.1673478

13. Berg RW, Leung E, Gough S, et al. Cloning and characterization of a novel beta integrin-related cDNA coding for the protein TIED ("ten beta integrin EGF-like repeat domains") that maps to chromosome band 13q33: a divergent stand-alone integrin stalk structure. Genomics. 1999;56(2):169-178. doi:10.1006/geno.1998.5707

14. Akhtar N, Streuli CH. An integrin-ILK-microtubule network orients cell polarity and lumen formation in glandular epithelium. Nat Cell Biol. 2013;15(1):17-27. doi:10.1038/ncb2646

15. Sun L, Wang D, Li X, Zhang L, Zhang H, Zhang Y. Extracellular matrix protein ITGBL1 promotes ovarian cancer cell migration and adhesion through Wnt/PCP signaling and FAK/SRC pathway. Biomed Pharmacother. 2016;81:145-151. doi:10.1016/j.biopha.2016. 03.053

16. Li XQ, Du X, Li DM, et al. ITGBL1 Is a Runx2 Transcriptional Target and Promotes Breast Cancer Bone Metastasis by Activating the TGFbeta Signaling Pathway. Cancer Res. 2015;75 (16):3302-3313. doi:10.1158/0008-5472.CAN-15-0240
17. Qiu X, Feng JR, Qiu J, et al. ITGBL1 promotes migration, invasion and predicts a poor prognosis in colorectal cancer. Biomed Pharmacother. 2018;104:172-180. doi:10.1016/j.biopha.2018.05.033

18. Matsuyama T, Ishikawa T, Takahashi N, et al. Transcriptomic expression profiling identifies ITGBL1, an epithelial to mesenchymal transition (EMT)-associated gene, is a promising recurrence prediction biomarker in colorectal cancer. Mol Cancer. 2019;18(1):19. doi:10.1186/s12943-019-0945-y

19. Li R, Zhuang C, Jiang S, et al. ITGBL1 Predicts a Poor Prognosis and Correlates EMT Phenotype in Gastric Cancer. $J$ Cancer. 2017;8 (18):3764-3773. doi:10.7150/jca.20900

20. Liu X, Wu J, Zhang D, et al. Identification of Potential Key Genes Associated With the Pathogenesis and Prognosis of Gastric Cancer Based on Integrated Bioinformatics Analysis. Front Genet. 2018;9:265. doi:10.3389/fgene.2018.00265

21. Xu MY, Qu Y, Li Z, Li F, Xiao CY, Lu LG. A 6 gene signature identifies the risk of developing cirrhosis in patients with chronic hepatitis B. Front Biosci. 2016;21:479-486. doi:10.2741/4403

22. Wang M, Gong Q, Zhang J, et al. Characterization of gene expression profiles in HBV-related liver fibrosis patients and identification of ITGBL1 as a key regulator of fibrogenesis. Sci Rep. 2017;7(1):43446. doi:10.1038/srep43446

23. Huang $\mathrm{W}$, Yu D, Wang M, et al. ITGBL1 promotes cell migration and invasion through stimulating the TGF-beta signalling pathway in hepatocellular carcinoma. Cell Prolif. 2020;53(7):e12836. doi:10. 1111/cpr.12836

24. Terrault NA, Lok ASF, McMahon BJ, et al. Update on prevention, diagnosis, and treatment of chronic hepatitis B: AASLD 2018 hepatitis B guidance. Hepatology. 2018;67(4):1560-1599. doi:10.1002/ hep. 29800

25. Marrero JA, Kulik LM, Sirlin CB, et al. Diagnosis, Staging, and Management of Hepatocellular Carcinoma: 2018 Practice Guidance by the American Association for the Study of Liver Diseases. Hepatology. 2018;68(2):723-750. doi:10.1002/hep.29913

26. Mazzaferro V, Bhoori S, Sposito C, et al. Milan criteria in liver transplantation for hepatocellular carcinoma: an evidence-based analysis of 15 years of experience. Liver Transpl. 2011;17(Suppl 2):S4457. doi:10.1002/lt.22365

27. Edge SB, Compton CC. The American Joint Committee on Cancer: the 7th edition of the AJCC cancer staging manual and the future of TNM. Ann Surg Oncol. 2010;17(6):1471-1474. doi:10.1245/s10434010-0985-4

28. Llovet JM, Brú C, Bruix J. Prognosis of hepatocellular carcinoma: the BCLC staging classification. Semin Liver Dis. 1999;19 (3):329-338. doi:10.1055/s-2007-1007122

29. Llovet JM, Di Bisceglie AM, Bruix J, et al. Design and endpoints of clinical trials in hepatocellular carcinoma. $J$ Natl Cancer Inst. 2008;100(10):698-711. doi:10.1093/jnci/djn134

30. Gan X, Liu Z, Tong B, Zhou J. Epigenetic downregulated ITGBL1 promotes non-small cell lung cancer cell invasion through Wnt/PCP signaling. Tumour Biol. 2016;37(2):1663-1669. doi:10.1007/s13277015-3919-8

31. Ji Q, Zhou L, Sui H, et al. Primary tumors release ITGBL1-rich extracellular vesicles to promote distal metastatic tumor growth through fibroblast-niche formation. Nat Commun. 2020;11(1):1211. doi:10.1038/s41467-020-14869-x

32. Galle PR, Foerster F, Kudo M, et al. Biology and significance of alpha-fetoprotein in hepatocellular carcinoma. Liver Int. 2019;39 (12):2214-2229. doi:10.1111/liv.14223

33. Kokudo N, Takemura N, Hasegawa K, et al. Clinical practice guidelines for hepatocellular carcinoma: the Japan Society of Hepatology 2017 (4th JSH-HCC guidelines) 2019 update. Hepatol Res. 2019;49 (10):1109-1113. doi:10.1111/hepr.13411 


\section{Publish your work in this journal}

The Journal of Hepatocellular Carcinoma is an international, peerreviewed, open access journal that offers a platform for the dissemination and study of clinical, translational and basic research findings in this rapidly developing field. Development in areas including, but not limited to, epidemiology, vaccination, hepatitis therapy, pathology and molecular tumor classification and prognostication are all considered for publication. The manuscript management system is completely online and includes a very quick and fair peer-review system, which is all easy to use. Visit http://www.dovepress.com testimonials.php to read real quotes from published authors. 